\title{
Primeiro Registro do Gênero e Espécie Neblinagena doylei Kodada \& Jäch (Coleoptera: Elmidae: Larainae) no Brasil
}

\author{
Jaime de Liege Gama Neto ${ }^{\bowtie} \&$ Mahedy Araujo Bastos Passos ${ }^{2}$
}

1. Universidade Estadual de Roraima, Museu Integrado de Roraima, e-mail: jaimebio@hotmail.com (Autor para correspondência ${ }^{\bowtie}$ ). 2. Universidade Estadual de Roraima, e-mail: mahedypassos@hotmail.com.

\section{EntomoBrasilis 9 (1): 62-64 (2016)}

\section{Registrado no ZooBank: urn:lsid:zoobank.org:pub:1E981356-5B6D-4A94-B37E-67D6C4A421D5}

Resumo. Registra-se pela primeira vez no Brasil Neblinagena doylei Kodada \& Jäch (Elmidae, Larainae) ampliando a distribuição geográfica do gênero e da espécie na América do Sul. São apresentadas informações sobre o ambiente e a qualidade da água do local de coleta da espécie. Adicionalmente, citam-se os seguintes gêneros e espécies de Elmidae amostradas juntamente com N. doylei: Cylloepus, Microcylloepus e Neoelmis e as espécies Xenelmis micros Grouvelle e Xenelmis tarsalis Hinton.

Palavras-Chave: América do Sul; Byrrhoidea; Coleópteros aquáticos; Distribuição geográfica; Elminae.

\section{First Record of Genus and Species Neblinagena doylei Kodada \& Jäch (Coleoptera: Elmidae: Larainae) in Brazil}

Abstract. We recorded, for the first time in Brazil, the genus and species Neblinagena doylei Kodada \& Jäch, expanding the geographic distribution of the genus and species in South America. We complement this information with data on the environment and water quality of the locality where the species was collected. Adittionally, we cited the following genera and species of Elmidae collected along with N. doylei: Cylloepus, Microcylloepus, Neoelmis, Xenelmis micros Grouvelle and Xenelmis tarsalis Hinton.

Keywords: Aquatic beetles; Byrrhoidea; Elminae; Geographic distribution; South America.

可 lmidae constitui uma ampla e diversa família de coleópteros aquáticos, com cerca de 1.330 espécies mundialmente conhecidas, das quais 148 são registradas para o Brasil (SEgURA et al. 2012). Atualmente é composta pelas sub-famílias, Elminae e Larainae sendo que a fauna brasileira de Larainae é praticamente desconhecida. Dos nove gêneros descritos em Larainae, há registros de ocorrência, no Brasil, de apenas seis espécies, distribuídas entre os gêneros: Hexanchorus, Phanocerus e Potamophilops (Segura et al. 2012; Manzo 2013). Hexanchorus ocorre no Espírito Santo [Hexanchorus thermarius (Coquerel)], Rio de Janeiro [Hexanchorus caraibus (Coquerel) e Hexanchorus gracilipes Sharp] e Santa Catarina (Hexanchorus tarsalis Hinton); Phanocerus clavicornis Sharp, tem registro de ocorrência no Amazonas, Rio de Janeiro e Santa Catarina, enquanto que Potamophilops cinereus Blanchard ocorre nos estados do Mato Grosso e São Paulo (Segura et al. 2012).

O objetivo deste trabalho é apresentar o primeiro registro do gênero e da espécie Neblinagena doyley Kodada \& Jäch no Brasil, mais precisamente no estado de Roraima, ampliando a sua distribuição geográfica e acrescentado informações sobre a ecologia e o hábitat da espécie. Adicionalmente, são apresentados outros gêneros e espécies de Elmidae amostrados juntamente com $N$. doylei, os quais representam novos registros de Elmidae para o estado de Roraima.

Os espécimes foram coletados de 09 a 12 de março de 2012, na serra do Tepequém, município de Amajari, Roraima, na área limítrofe entre a Venezuela e o Brasil (03 $83^{\circ} 39.6 ” \mathrm{~N} / 61^{\circ}$
44'49.8” W) (Figura 1). A serra do Tepequém possui altitude máxima de aproximadamente $1.140 \mathrm{~m}$ e faz parte de um conjunto de blocos rochosos de forma tabular que ocorrem dentro e nas proximidades da "Gran Sabana" venezuelana (Figura 2A), conhecidos localmente pelo termo "tepui" (BARBOSA 1992) e está concentrada em uma zona de transição entre campos e floresta tropical densa (Melo \& Filho 1996).

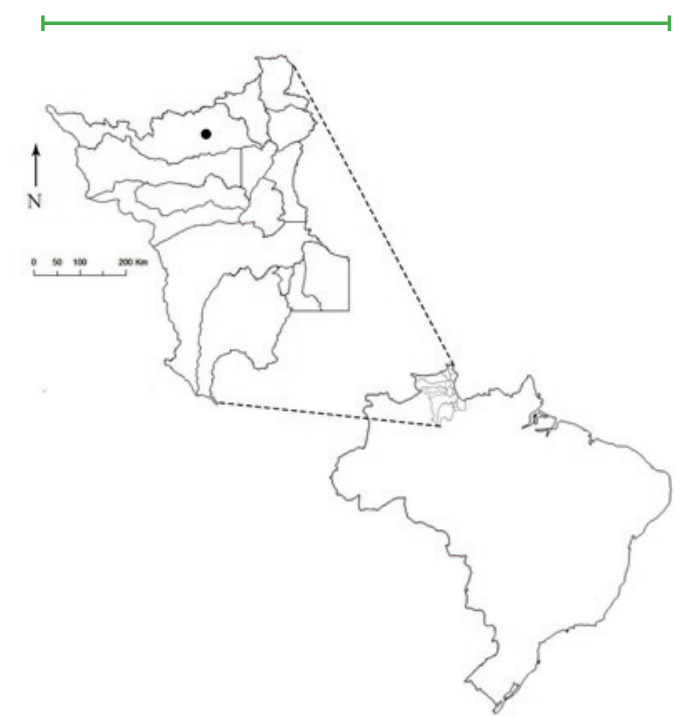

Figura 1. Mapa com localização da serra do Tepequém no estado de Roraima, Brasil. 

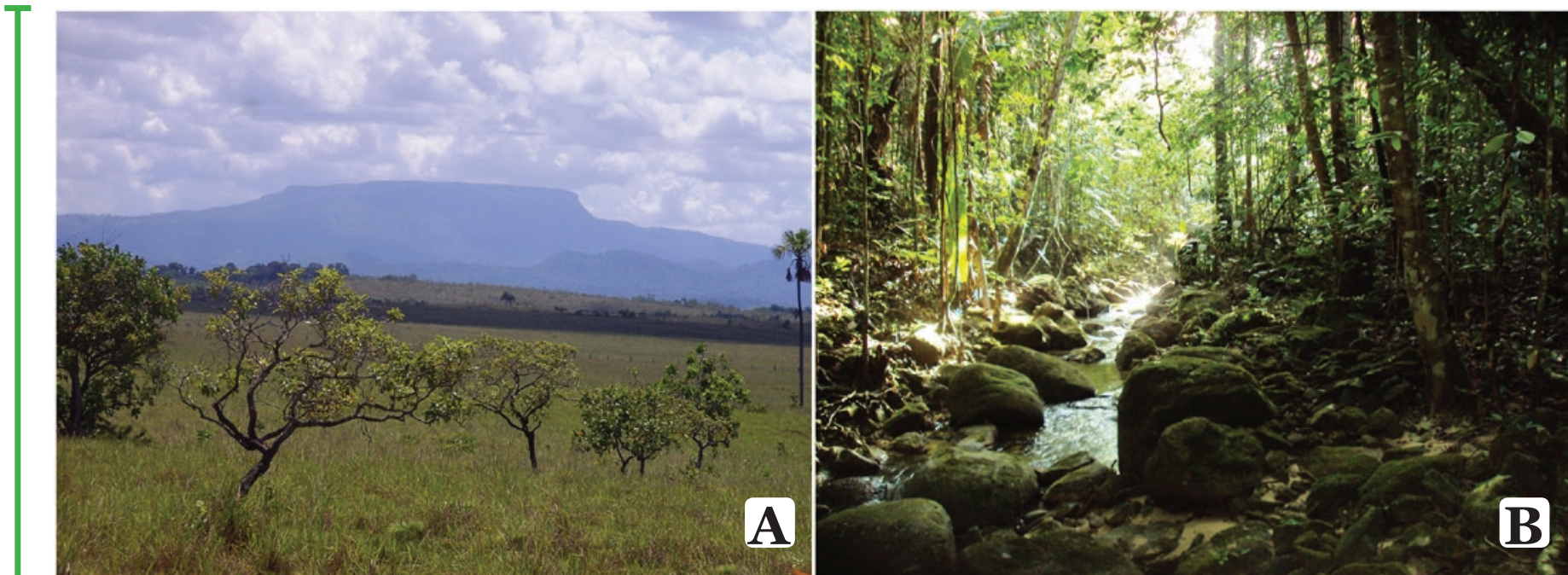

Figura 2. Visão geral do local de coleta: A. Serra do Tepequém; B. igarapé onde Neblinagena doylei Kodada \& Jäch (Coleoptera, Elmidae) foi coletada.

As coletas foram realizadas com auxílio de armadilha luminosa tipo Pensilvânia e rede "D”, em um igarapé de pequena ordem, caracterizado por apresentar as margens bem protegidas por uma vegetação ripária densa que propicia cobertura vegetal sobre o leito. O fundo do igarapé é rochoso, constituído predominantemente por áreas de corredeira, com profundidade média inferior a $50 \mathrm{~cm}$ na época seca, estando sujeito a variações diárias no período chuvoso, devido à enxurradas que elevam rapidamente a profundidade para aproximadamente $1,5 \mathrm{~m}$ no momento das chuvas, retornando ao nível inicial com o cessar das mesmas (Figura 2B). No momento das coletas a temperatura da água foi de $23,3{ }^{\circ} \mathrm{C}$, com pH levemente ácido $(6,8)$, elevados níveis de oxigênio dissolvido (84\%) e condutividade elétrica de $11,8 \mu \mathrm{mS} / \mathrm{cm}$.

A identificação das espécies foi realizada com base em KodADA \& Jäch (1999), Manzo (2005) e MaIER (2013). A fotografia de $N$. doylei foi realizada com auxílio de um estereomiscroscópio Olympus SZ61 e de uma máquina fotográfica digital FUJI Fine Pix S-4000 acoplada à ocular. Todo o material coletado encontrase depositado na coleção de entomologia do Museu Integrado de Roraima - MIRR.

O gênero Neotropical Neblinagena é endêmico da Venezuela e facilmente diferenciado dos demais gêneros de Larainae pelo seu tamanho relativamente grande (5,5 - 6,6 mm de comprimento), corpo delgado de coloração escura, pronoto distinto, com uma carena lateral longitudinal ou com sulco sinuoso-arqueado no terço basal e pelas duas carenas pré-escutelares curtas e convergentes, ambas com uma fossa lateral profunda (Figura 3).

O gênero apresenta apenas duas espécies descritas (Neblinagena prima Spangler e $N$. doylei), encontradas em regiões de altitudes elevadas da Venezuela ( $>400 \mathrm{~m}$ ), em pequenos rios com rochas e cobertura vegetal. A espécie $N$. doylei pode ser distinguida de $N$. prima pela carena pronotal longitudinal que é inteira e pelo sulco oblíquo do pronoto formando um ângulo de $30^{\circ}$ em relação ao sulco longitudinal mediano (Figura 3), enquanto que em $N$. prima a carena pronotal longitudinal é anteriormente obscura e o sulco oblíquo do pronoto forma um ângulo de $45^{\circ}$ em relação ao sulco longitudinal mediano; adicionalmente, o tamanho do corpo em $N$. doylei é relativamente mais largo que em $N$. prima (MAIER 2013).

Material examinado: Neblinagena doylei. BRASIL. 27 machos. Município de Amajari, Serra do Tepequém, estado de Roraima. Igarapé de segunda ordem (03 $\left.{ }^{\circ} 87^{\prime} 39.6 ” \mathrm{~N} / 61^{\circ} 44^{\prime} 49.8^{\prime \prime} \mathrm{W}\right)$. Altitude $551 \mathrm{~m}$ acima do nível do mar. 09-12.iii.2012, Gama Neto, J.L. coll.
Na Venezuela, $N$. doylei tem sido encontrada nas regiões da Gran Sabana e La Escalera, agarrada a pedras planas, sob a lâmina d'água, associada à Roraima carinata Kodada \& Jäch [(Elmidae, Elminae) espécie endêmica do Monte Roraima] e à elmídeos do gênero Macrelmis (MAIER 2013). No Brasil, o habitat no qual $N$. doylei foi coletada corresponde àquele já descrito para o gênero, em igarapé pequeno, com altitude superior a $400 \mathrm{~m}$ acima do nível mar, rochoso e com relativa cobertura vegetal. Na serra do Tepequém, a espécie foi observada saindo debaixo de pedras rugosas que se encontravam parcialmente submersas, voando por pouco tempo sob a lâmina d'água e retornando novamente para a parte submersa das rochas. No momento em que voavam sobre a lâmina d'água era possível coletar os espécimes com o auxílio de uma rede " $\mathrm{D}$ ".

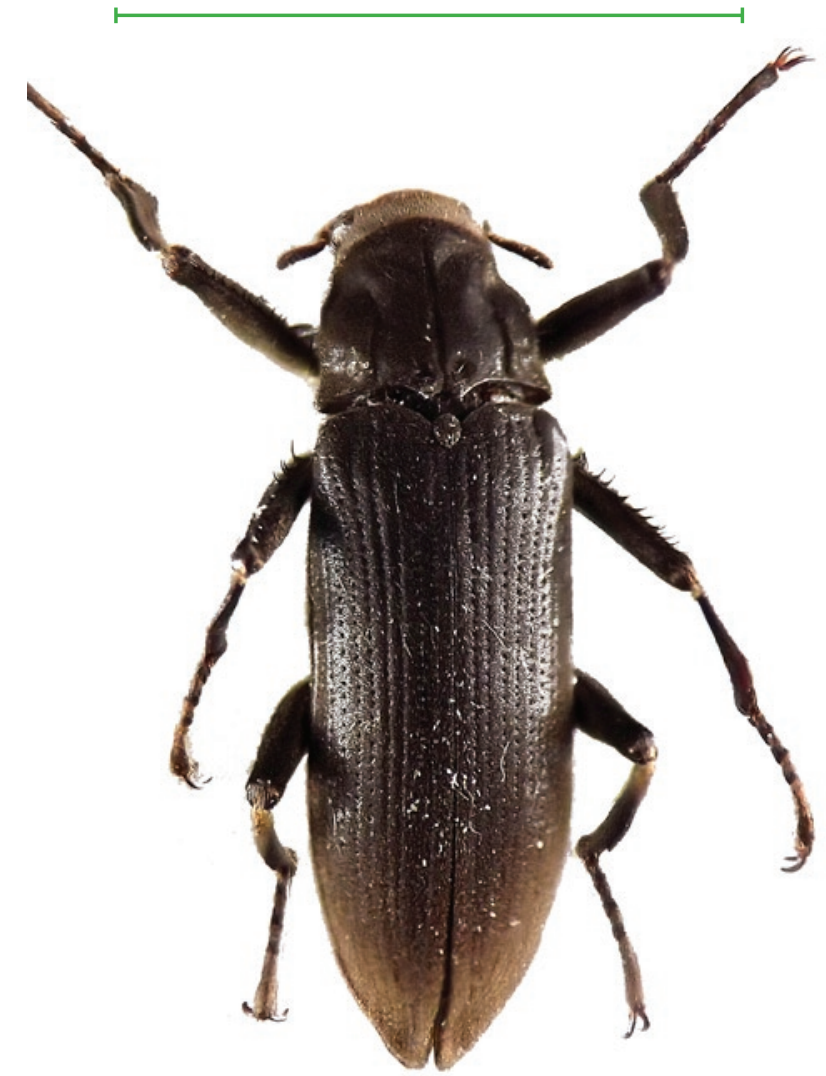

Figura 3. Neblinagena doylei Kodada \& Jäch (hábito dorsal).

No local amostrado, além de N. doylei, também foram coletados elmídeos pertencentes aos gêneros Cylloepus, Macrelmis, Microcylloepus e Neoelmis, além de Xenelmis micros Grouvelle e Xenelmis tarsalis Hinton (Coleoptera: Elmidae: Elminae), 
todos representando novos registros de ocorrência para o estado de Roraima.

\section{REFERÊNCIAS}

Barbosa, R.I., 1992. Um Tepui no ritmo da destruição em Roraima. Ciência Hoje 14: 94-96.

Kodada, J. \& M.A., Jäch, 1999. Roraima carinata gen. et sp. nov. and Neblinagena doylei sp. nov., two Larainae from Mount Roraima, Venezuela (Coleoptera: Elmidae). Entomological Problems, 30: 13-30.

Maier, C.A., 2013. A revision of the Larainae (Coleoptera, Elmidae) of Venezuela, with description of nine new species. ZooKeys, 329: 33-91. DOI: http://dx.doi.org/10.3897/ zookeys.329.4961.

Manzo, V., 2005. Key to the South America genera of Elmidae (Insecta: Coleoptera) with distributional data. Studies on Neotropical Fauna and Environment, 40: 201-208.
Manzo, V., 2013. Los élmidos de la región Neotropical (Coleoptera: Byrrhoidea: Elmidae): diversidad y distribución. Revista de la Sociedad Entomológica Argentina, 72: 199-212.

Melo, E.C. \& R.A. Filho, 1996. Mapeamento de áreas degradadas pela atividade de garimpos na região da serra Tepequém (RR), através de imagens Landsat-TM. Anais VIII Simpósio Brasileiro de Sensoriamento Remoto, INPE. 639-645.

Segura, M.O., Valente-Neto, F. \& A.A. Fonseca-Gessner, 2012. Checklist of the Elmidae (Coleoptera: Byrrhoidea) of Brazil. Zootaxa, 3260: 1-18.

\section{Recebido em: o6.ix.2015}

Aceito em: 14.xii.2015

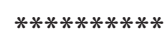

\section{Como citar este artigo:}

Gama Neto, J.L. \& M.A.B. Passos, 2016. Primeiro Registro do Gênero e Espécie Neblinagena doylei Kodada \& Jäch (Coleoptera: Elmidae: Larainae) no Brasil. EntomoBrasilis, 9 (1): 62-64.

Acessível em: doi:10.12741/ebrasilis.vgi1.550
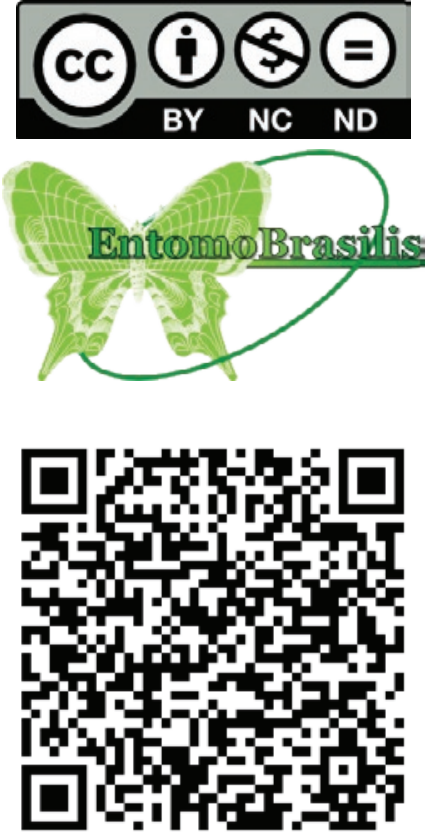